\title{
Recent advances in beta decay measurements
}

\author{
Magali Estienne*, Muriel Fallot, Lydie Giot, Loïc Le Meur, and Amanda Porta \\ Subatech (CNRS/IN2P3), IMT Atlantique, Université de Nantes, 4 Rue Alfred Kastler, 44307 Nantes, France
}

Received: 20 November 2017 / Received in final form: 15 February 2018 / Accepted: 18 May 2018

\begin{abstract}
Three observables of interest for present and future reactors depend on the $\beta$ decay data of the fission products: the reactor decay heat, antineutrinos from reactors and delayed neutron emission. Concerning the decay heat, significant discrepancies still exist between summation calculations in - their two main ingredients: the decay data and the fission yields - performed using the most recent evaluated databases available. It has been recently shown that the associated uncertainties are dominated by the ones on the decay data. But the results subtantially differ taking into account or not the correlations between the fission products. So far the uncertainty propagation does not include as well systematic effects on nuclear data such as the Pandemonium effect which impacts a large number of nuclei contributing to the decay heat. The list of nuclei deserving new TAGS measurements has been updated recently in the frame of IAEA working groups. The issues listed above impact in the same way the predicted energy spectra of the antineutrinos from reactors computed with the summation method, the interest of which has been recently reinforced by the Daya Bay latest publication. Nuclear data should definitely contribute to refine and better control these calculations. Lastly, a lot of nuclear data related to delayed neutrons are missing in nuclear databases. Despite the progresses already done these last years with new measurements now requiring to be included in evaluated databases, the experimental efforts which still need to be done are significant. These different issues will be addressed here before to comment on recent experimental results and on their impacts on the quoted observables. Some perspectives will also be presented. Solving the issues listed above will require to bring together experimental, simulation, evaluation and theoretical activities.
\end{abstract}

\section{Introduction}

In Pressurized Water Reactors, the thermal power is mainly induced by four isotopes constituting the fuel assembly. The two fissile and fertile nuclei ${ }^{235} \mathrm{U}$ and ${ }^{238} \mathrm{U}$ present in the fresh fuel undergo fission or capture processes inducing the production of the fissile nuclei ${ }^{239} \mathrm{Pu}$ and ${ }^{241} \mathrm{Pu}$. The evolution of their compositions with the time $\mathrm{t}$ (reactor burnup) is typical: while the ${ }^{235} \mathrm{U}$ composition dominantly decreases with t compared to ${ }^{238} \mathrm{U},{ }^{239} \mathrm{Pu}$ is massively produced in comparison to ${ }^{241} \mathrm{Pu}$. In the fission process which gives the thermal energy according to equation (1), we are essentially interested by the production of the two fission products FP1 and FP2.

$$
n+{ }^{235} \mathrm{U} \rightarrow{ }^{236} \mathrm{U}^{*} \rightarrow F P 1+F P 2+\text { neutrons. }
$$

These latters most oftenly neutron-rich nuclei undergo $\beta$ or $\beta$-n decays (when the daughter nucleus is produced in an excited state above the neutron emission threshold $S_{n}$ ). In such radioactive processes, a neutron converts into a

\footnotetext{
* e-mail: magali.estienne@subatech.in2p3.fr
}

proton and emits an electron and an electron antineutrino. Gammas are also emitted in the deexcitation of the produced daughter. Their independent measurement allows to assess $\beta$ decay physics properties. In these proceedings, we will essentially focus on the physics that can be addressed via the $\gamma$ channel.

Before the $90 \mathrm{~s}$, high-resolution $\gamma$-ray spectroscopy through the use of germanium detectors was the conventional detection technique used for $\gamma$ measurement. It offers excellent energy resolution but an efficiency which strongly decreases at high energy and usually limited acceptance. As direct consequences, weak $\gamma$-ray cascades or high energy $\gamma$-rays are likely difficult to detect and the decay scheme of the parent nucleus may be incomplete. There is a danger of overlooking the existence of $\beta$-feeding into the high energy nuclear levels of daugther nuclei especially with decay schemes with large $Q$-values. In other words, it might lead to an overestimate of the high energy part of the FP $\beta$ spectra along with an underestimate of the $\gamma$ energies. This phenomenon is commonly called "Pandemonium effect" highlighted by Hardy [1]. As a consequence, the content of nuclear databases (NDB) can be strongly biased in some cases, as illustrated in Figure 1 in reference [2]. In this figure, two antineutrino energy spectra reconstructed from 
the endpoints and branching ratios either tabulated in JEFF 3.1 or measured by the TAGS collaboration (Pandemonium free - see Sect. 3.1 for details on the detection technique) for ${ }^{105} \mathrm{Mo}$ are compared. The average energy of $1.524 \mathrm{MeV}$ of the latter is to be compared to the $2.475 \mathrm{MeV}$ of the former one. Their difference of the order of $50 \%$ is to be compared to error tabulated in JEFF of the order of the percent. The uncertainty has thus been clearly underestimated in this case. This illustrates the necessity to manipulate and use NDB with the utmost care.

Indeed $\beta$ decay has important implications for present and future reactors. They are threefold: (i) The released $\gamma$-ray and $\beta$ particle contribute to the decay heat (DH) which is critical for reactor safety and economy; (ii) The antineutrinos which escape can be detected. This impacts both fundamental physics (neutrino oscillation, mass hierarchy and reactor anomaly) and reactor applications (monitoring and non-proliferation); (iii) The study of $\beta$-n emitters provides an access to the delayed neutron fractions important for the operation and control of the chain reactions of reactors. In Section 2, the current status of the researchs carried out in those branches will be given and analyzed in the point of view of nuclear data. Specific attention will be focused on the treatment of uncertainties. Section 3 will be devoted to a discussion on recent and expected new results from the TAGS collaboration. At this occasion, the TAGS detection technique will be presented. Our conclusions and perspectives will be given in Section 4 .

\section{Current status of $\beta$ decay applications}

\subsection{Decay heat}

The decay heat (DH) is defined as the energy released by the nuclear fuel after the shut-down of the chain reaction in a reactor. It concerns the three $\alpha, \beta$ and $\gamma$ radioactivities whose emitters are either the FPs (which are the dominant contributors to the DH over the first 100 years after the reactor stop) or heavy nuclei such as the actinides (which contribution becomes not negligeable above 100 years after the reactor stop). As the $\mathrm{DH}$ represents a residual power of 6 to $12 \%$ of the nominal power of the reactor just after its shutdown, the evaluation of the reaction safety and various economic aspects of nuclear power generation require its good knowledge. The DH can be directly accessed and studied via integral measurements (for a recent review see [3]). However, it is also essential to be able to estimate it analytically and this is achievable thanks to the only predictive method for future reactors: the summation method [4]. It consists in the summation of all the fission product and actinide contributions inventoried for specific conditions of reactor operation and subsequent cooling period. In the case of fission pulses, the time ranges up to $\sim 10^{6} \mathrm{~s}$ and the fission of pure isotopes is considered. In these conditions, the contribution of actinides can be neglected and the decay heat can be expressed with equation (2),

$$
f(t)=\sum_{i}\left(\bar{E}_{\beta, i}+\bar{E}_{\gamma, i}\right) \lambda_{i} N_{i}(t)
$$

where $\bar{E}_{\beta, i}$ and $\bar{E}_{\gamma, i}$ are respectively the mean energies of the $\beta$ and $\gamma$ decays of the ith FP, $\lambda_{i}$ is its total decay constant and $N_{i}(t)$ the associated fission yield. The computation thus requires the access to these observables in NDB for as much fission products as possible with the best accuracy as possible. In the 70s, this formula was successfully computed with the use of nuclear DB taken worldwide, however important discrepancies were observed comparing the DH calculations and benchmark experiments mainly because of the Pandemonium effect. These differences were partially compensated by the inclusion of average $\beta$ and $\gamma$ energies derived from the Gross Theory of $\beta$ decay which was able to compensate the missing $\beta$-strengths or the missing nuclei information $[5,6]$. But, since then this temporary solution was step-by-step replaced by the use of measured data with a new detection technique: the total absorption $\gamma$-ray spectroscopy TAGS that will be presented in Section 3.1. Since then, several collaborations and some worldwide efforts around TAGS are born with the support of international agencies as the IAEA, the NEA, etc. Figure 2 in reference [7] highlights how the use of non Pandemonium free measurements stored in NDB can strongly bias the computation of the DH and the success of the TAGS to correct for this effect. During the 1st TAGS campain of the Valencian's group of IFIC at the facility of JYFL of Jÿvaskÿla (Finland), Algora et al. measured the $\beta$-feeding of the 7 nuclei $102 ; 104 ; 105 ; 106 ; 107 \mathrm{Tc},{ }^{105} \mathrm{Mo}$, and ${ }^{101} \mathrm{Nb}$ important contributors to the DH, 5 out of 7 indeed were suffering strongly from Pandemonium effect. The summation calculation of the DH has then been repeated substituting their measurements to the ones previously stored in ENDF/B-VII which came from high resolution measurements. These nuclei strongly contributed to improve the calculation reducing impressively the gap between Tobias integral measurement compilation [8] and the summation calculation and solving a large part of the long-standing discrepancy in the $\gamma$ component of the decay-heat data of ${ }^{239} \mathrm{Pu}$ in the $4-3000$ s range.

Figure 1 presents a comparison of some FISPACT-II simulations of the total and the electromagnetic decay heats performed with a variety of nuclear databases (ENDF/B [9], JEFF [10] and JENDL [11], the American, European and Japanese libraries respectively containing neutron-induced fission yield (nFY) and a variety of decay data (DD) and the General Description of Fission Observables model (GEF) [12]) to integral measurements on a thermal fission of ${ }^{235} \mathrm{U}$ [3]. The measurements performed from fission decay heat experiments include the ones using the Oak Ridge Research Reactor by Dickens et al. [13], a series of experiments performed under the direction of Schier from the university of Massachusetts Lowell [14-16] and a separate meta-analysis performed by Tobias which includes a combination of results from multiple laboratories [8]. Even if this set of comparative studies demonstrates the ability to calculate the decay heat within the experimental uncertainties of the various integral measurements, some efforts still need to be done in order to improve the quality of both nuclear databases and integral measurements to reach agreements between simulations and experiments. As an example the three following observations can be made regarding the $\gamma$ component of the $\mathrm{DH}$ of ${ }^{235} \mathrm{U}$ : i) Integral measurements 


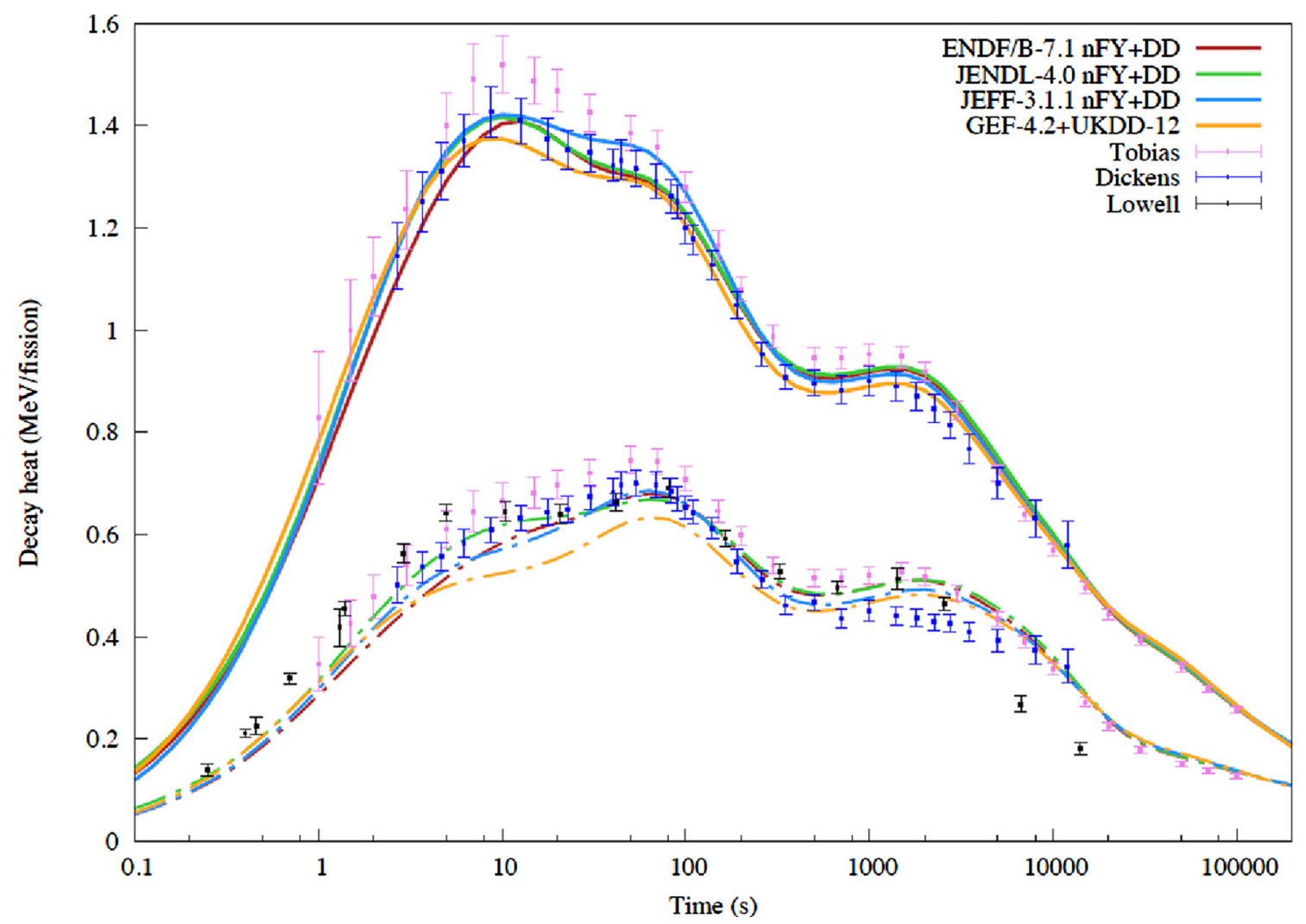

Fig. 1. Total (solid) and $\gamma$ (dash) decay heat from thermal pulse on ${ }^{235} \mathrm{U}$ as a function of time (s) [3].

are not in agreement for several cooling times of the most well-known nuclide; ii) There are still discrepancies between data and simulations using different DB for $\gamma$ heat; iii) The comparison between data and simulations is better for the total heat than for the $\gamma$ one in favour of potential problems in the decay data $\beta / \gamma$ feeds.

Concerning the determination of the total uncertainty associated to the calculation of the $\mathrm{DH}$, a lot of work still needs to be done to improve our knowledge about $\beta$ decay properties of FP and to estimate the associated uncertainties. In his $\mathrm{PhD}$ thesis, Benoit concludes that the uncertainty on its decay heat calculation mainly comes from the uncertainties on the fission yields [17] in opposition to the work performed by Katakura which points out that the main contribution to decay heat uncertainties comes from the evaluation of decay energies below $5 \times 10^{3} \mathrm{~s}[18]$. Concerning the mean decay energy in the first approach [17], a mean error of $3.35 \%$ was considered for 369 nuclei tabulated in JEFF3.1, and 10\% of uncertainties have been taken for 75 unknown nuclei. These are mainly nuclei with half life $<10$ s. Whereas in the second approach [18] (probably more realistic having in mind the bias coming from Pandemonium effect in nuclear databases discussed in Sect. 1) 100\% error was considered on nuclei for which no uncertainty was given in databases. However, a really low uncertainty of $0.001 \%$ on 122 branching ratios over 250 has been considered in the first approach and no sensitivity study has been performed on the BRs in the second approach leaving an open question about the impact of uncertainties on BRs. Moreover different covariant matrices of the fission yields have been included in both works showing the necessity to take them into account in the complete computation of the $\mathrm{DH}$ uncertainty and emphasizing the need for some additional reflection on how to compute the covariance matrices.

\subsection{Reactor antineutrinos}

Reactor antineutrinos are a second direct application of $\beta$ decay both from fundamental and applied point of views.

In the field of the fundamental physics of neutrinos, the last 5 years have been essential for the first measurements of the $\theta_{13}$ mixing angle by the three experiments Double Chooz (DC) [19], Daya Bay (DBay) [20] and RENO [21]. Indeed, the precise measurement of the oscillation parameter required an independent computation of the antineutrino spectra. The conversion method, quasi independent from nuclear databases and in principle more precise than the summation method was chosen at the time by the different experiments for that purpose [22].

The spectral studies carried out for $\theta_{13}$ determination are at the origin of the so-called "reactor anomaly" [23]. They are responsible for the $6 \%$ deficit of the absolute value of the measured flux compared to the best prediction converted ILL data which has been first observed by short distance experiments and later confirmed by DC, DBay and Reno. One of the hypotheses which has been proposed to explain this deficit was the potential existence of one or several sterile neutrino(s), hypothesis which has motivated the deployments of a large amount of short baseline 
experiments [24]. However, an other hypothesis that could be considered concerns the converted spectra and to which extent they can be regarded as trustful. This hypothesis should not be neglected taking into account that: i) The three experiments observed a distortion in their full antineutrino energy spectra between 5 and $7 \mathrm{MeV}$ not reproduced so far by the converted spectra; ii) The last results from DBay which points-out a potential problem in the converted antineutrino spectra from ${ }^{235} \mathrm{U}$ measured $\beta$ spectrum at ILL $[25,26]$. The quite large number of recent publications on spectral studies in the frame of neutrino physics putting into question the integral $\beta$ measurement of ${ }^{235} \mathrm{U}$ of Schreckenbach et al. and its conversion and the potential existence of sterile neutrinos strongly motivate the necessity to improve nuclear databases used by the summation method, the alternative to the conversion one [26]. Antineutrino spectra predictions will also be essential for the next generation reactor neutrino experiments like JUNO [27] or to compute the background for other multipurpose experiments.

As regard neutrinos and applied physics, it has been suggested to use the discrepancy between antineutrino flux and energies from $\mathrm{U}$ and $\mathrm{Pu}$ isotopes to infer reactor fuel isotopic composition and power [28]. Indeed, due to the nuclear structure properties of the 4 isotopes present in a reactor core, the distribution of their fission products is different implying different antineutrino emitted energy spectra after they undergo a $\beta$ decay. On an average 6 antineutrinos are produced per fission and about 2 above the inverse $\beta$ decay (IBD) reaction threshold, the reaction commonly used for their detection. Moreover, due to the time evolution of the fuel isotopic composition, the associated antineutrino flux also evolves with time both in norm and shape making of antineutrino detection an elegant tool to monitor the core fissile content. This second topic associated to neutrinos strongly motivates the necessity to perform new measurements of nuclei (of interest for the field) which $\beta$ decay. The IAEA Nuclear Data Section has acknowledged this necessity by including a number of nuclei of interest for reactor antineutrino spectra in their priority lists of measurements [29].

\subsubsection{The summation method}

As for the decay heat, the antineutrino spectrum associated with one of the four fissioning isotopes in a moderated reactor can be computed as the sum of the contributions of all fission products using the full information available per nucleus in the NDB $[22,30]$. This method is useful for several reasons. Not only it is the only one that can be adapted to the computation of the antineutrino $(\bar{v})$ emission associated to various reactor designs, but also it allows for the computation of antineutrino spectra for which no integral $\beta$ spectrum has been measured yet taking into account off-equilibrium effects and allowing the use of different energy binnings of interest for reactor neutrino experimental analyses.

The $\beta / \bar{v}$ spectrum per fission of a fissible isotope $k$, $S_{k}(E)$, can be broken-up into the sum of all fission product $\beta / \bar{v}$ spectra weighted by their activity $A_{f p}$ according to equation (3). The $\beta / \bar{v}$ spectrum of one fission product being defined as the sum over the $b$ branches of all $\beta$ decay spectra (or associated $\bar{v}$ spectra), $S_{f p}^{b}$, of the parent nucleus to the daughter nucleus weighted by their respective branching ratios.

$$
S_{k}(E)=\sum_{f p=1}^{N_{f p}} A_{f p} \times \sum_{b=1}^{N_{b}} B R_{f p}^{b} \times S_{f p}^{b}\left(Z_{f p}, A_{f p}, E_{0 f p}^{b}, E\right) .
$$

To calculate these quantities the MURE code is used to extract the percentage of fissions and the fission product activity [31]. The $\beta$ spectrum $S_{f p}^{b}$ of each $b$ branch for the $\mathrm{fp}^{\text {th }}$ fission product is derived from the Fermi theory taking into account Huber's prescriptions for the treatment of corrections to be applied to the calculation [32]. Equation (3) illustrates the strong dependence of the summation method to nuclear data as $B R_{f p}^{b}, Z_{f p}, A_{f p}$ and $E_{0 f p}^{b}$ are extracted from NDB for each spectral prediction as described in [30]. A given spectral computation thus requires the use of databases as exhaustive and accurate as possible, including existing measurements Pandemonium free. It also motivates the necessity to perform new measurements of nuclei with incomplete decay schemes for which the $\beta$-feeding is clearly biased as explained in Section 1.

\subsubsection{Results after Pandemonium bias correction}

Figure 2 shows our predictions obtained in 2012 with the summation method of the antineutrino energy spectra emitted by the ${ }^{239} \mathrm{Pu}$ (left) and ${ }^{235} \mathrm{U}$ (right) in a PWR. The details of such computation can be found elsewhere [30]. We emphasize here that to obtain these new spectra, we substituted 7 nuclei, ${ }^{102,104,105,106,107} \mathrm{Tc},{ }^{101} \mathrm{Nb}$ and ${ }^{105} \mathrm{Mo}$, first simulated with the JEFF DB by the ones, Pandemonium free measured during the first TAGS campaign at the JYFL of Jÿvaskÿla (same nuclei as in Sect. 2.1). In the corresponding publication, the impact of these nuclei on the predicted spectra has been quantified and commented by looking at the ratio of the new predicted spectra and the ones obtained with the same data set but the latest TAGS data. The Pandemonium effect strongly affects the global $\bar{v}$ energy spectra of ${ }^{235,238} \mathrm{U}$ and ${ }^{239,241} \mathrm{Pu}$. A noticeable deviation from unity (maximum $8 \%$ decrease) was obtained for ${ }^{239,241} \mathrm{Pu}$. We observed a maximum of $3.5 \%$ deviation for ${ }^{238} \mathrm{U}$. As regards ${ }^{235} \mathrm{U}$, as these nuclei have a small contribution to the total spectrum, the effect was smaller than $1.5 \%$ at $\sim 3 \mathrm{MeV}$.

In these proceedings we would like to highlight the two inserts included in both panels of Figure 2 which correspond to the ratios of the spectra predicted with the summation method over the spectra predicted by the conversion one for ${ }^{239} \mathrm{Pu}$ (left) and ${ }^{235} \mathrm{U}$ (right). This powerful comparison with the computations of Huber over the range $2-8 \mathrm{MeV}$ shows reasonable agreement in terms of normalization and shape, despite the steeply falling shape of the spectra with energy. However, we observe a small increasing trend (reaching at maximum 10\% discrepancy up to $7 \mathrm{MeV}$ ) of the ratio with energy for ${ }^{239} \mathrm{Pu}$ suggesting that the NDB used are not yet fully Pandemonium free. This strongly motivates the necessity to measure more 


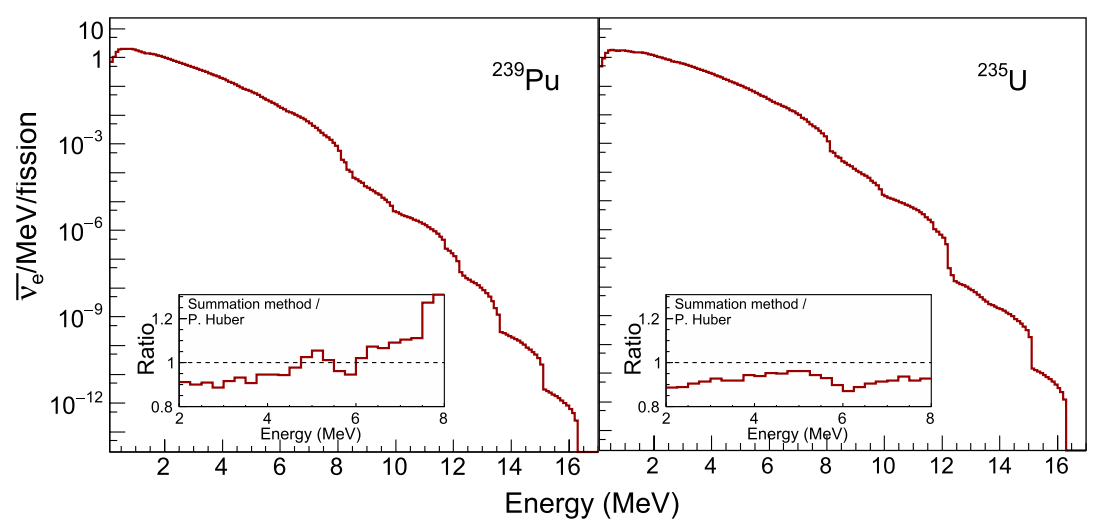

Fig. 2. Reconstructed antineutrino energy spectra, including the latest TAGS data from [7] for ${ }^{239} \mathrm{Pu}$ (left) and ${ }^{235} \mathrm{U}$ (right). In the insets are the ratios of the spectra to the ones computed by Huber (converted spectra) [30].

Pandemonium nuclei with the TAGS technique. Moreover for ${ }^{235} \mathrm{U}$, we obtained a ratio below 1 over the full energy range varying from less than $5 \%$ to $10 \%$ discrepancies. This observation is compatible with the latest PRL from Daya Bay which pointed out a potential problem in the converted spectrum of ${ }^{235} \mathrm{U}$ and supports the necessity to perfect our summation predictions with cleaner nuclear data.

At last, we would also like to stress the necessity to put uncertainties on our predictions which would definitively allow us to draw quantitative conclusions on comparison to neutrino experiments. For that purpose, one would need to have covariances between fission yields but also covariances for the decay data.

In [33], besides publishing our priority list of Pandemonium nuclei to be measured with TAGS, we have studied the impact of the recently measured ${ }^{92} \mathrm{Rb}$ on the decay heat and the antineutrino energy spectra of ${ }^{235,238} \mathrm{U}$ and ${ }^{239,241} \mathrm{Pu}$. Figure 3 illustrates the results of this new measurement on the spectra by comparing the ratio of the new predicted spectra and the ones obtained with the same data set but the latest TAGS data for ${ }^{92} \mathrm{Rb}$ (red dasheddotted line). As expected, the main effect is in the 4 to $8 \mathrm{MeV}$ antineutrino energy range, with a maximum between 7 and $8 \mathrm{MeV}$, and amounts to $4.5 \%$ for ${ }^{235} \mathrm{U}$, $3.5 \%$ for ${ }^{239} \mathrm{Pu}, 2 \%$ for ${ }^{241} \mathrm{Pu}$ and $1.5 \%$ for ${ }^{238} \mathrm{U}$. These discrepancies are due to the difference in the shapes of the antineutrino spectra built with the newly measured $\beta$ feedings with respect to the antineutrino spectra converted from Rudstam's measurements. The ratio is displayed as well with green dotted lines, and is nearly superposed on the ratio built when using Rudstam data in the first place. The change becomes even more dramatic (black dashed lines) if one compares with summation method spectra in which an older version of the ENDF data was used, as in [35]. A gray horizontal bar is placed above the antineutrino energy scale to indicate the region of the distortion observed by the reactor antineutrino experiments with respect to converted spectra. This shows the relevance of the present ${ }^{92} \mathrm{Rb}$ decay data in the calculations. It also motivates the needs for more measurements to improve DB and to precisely quantify the uncertainties associated to the summation calculations.

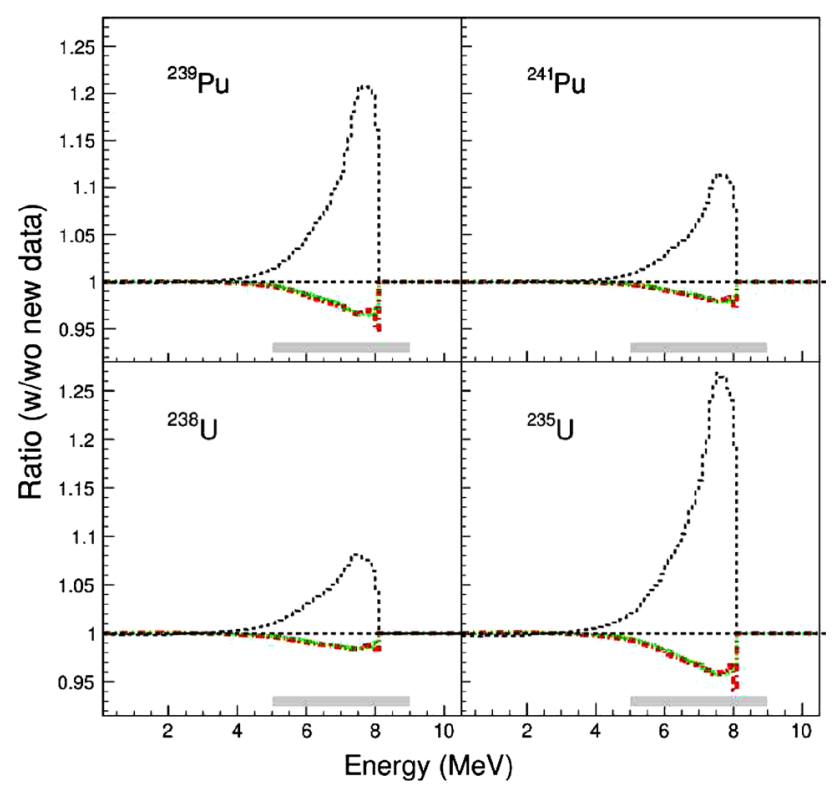

Fig. 3. Ratio between the antineutrino spectra calculated using the results presented in [33] with respect to the data on ${ }^{92} \mathrm{Rb}$ decay used in [30] (thick red dashed-dotted line), in [34] (green dotted line) and in [35] (black dashed line) for ${ }^{239} \mathrm{Pu}$ (top left), ${ }^{241} \mathrm{Pu}$ (top right), ${ }^{238} \mathrm{U}$ (bottom left) and ${ }^{235} \mathrm{U}$ (bottom right).

\section{$2.3 \beta$-Delayed neutrons (BDN)}

In some $\beta$ decays, if the daugther nucleus is produced in an excited state of energy above the neutron emission threshold, the corresponding nuclei can deexcite with the emission of a neutron producing a new nucleus of characteristics $(Z+1, N-2)$. This neutron is called delayed neutron with respect to the fast neutrons emitted during the fission process. A good control of these $\beta$-delayed neutron emission being essential for the reactor operation, it is crucial to have access to all the parameters characterizing a $\beta$-delayed neutron emission ideally via a dedicated database. Indeed the last compilation of $\beta$-delayed neutron data was published in 2002. Since then, many measurements were performed and published but no 
effort had been dedicated to list the whole data available worldwide. This has become an other concern of the IAEA which nuclear data section has organized a coordinated research project on the topic, with the aim of making a new database with up to date data of delayed neutron emission [36] and the aim of deducing from this new compilation a list of recommandations for new experimental efforts.

In addition, the IAEA has organized regular TAGS consultant meetings during the last decades [29], allowing to update the table of priority nuclei for the decay heat calculations and the reactor antineutrino predictions. The most recent table has been published in [33], and combines the important contributors to the decay heat, reactor antineutrinos, mentioning if they are $\beta$-delayed neutron emitters.

In parallel, the CEA Cadarache has started an experimental program in order to complement the databases in neutron delayed emission data [37].

\section{Recent and upcoming results in the European TAGS Collaboration}

\subsection{The total absorption spectroscopy: a solution to the Pandemonium effect}

In order to avoid the detection issue pointed-out in Section 1 regarding the use of HPGe detectors to measure $\gamma$-ray emissions, the Total Absorption $\gamma$-ray Spectroscopy (TAGS) technique was proposed in the 90s and successfully used since then to detect $\beta$ intensity to states at high excitation in the daughter nucleus [38,39]. A total absorption spectrometer (TAS) consists in a $\sim 4 \pi$ calorimeter for the detection of the $\gamma$ cascades rather than individual $\gamma$-rays. The detection of the total energy allows the determination of the feeding probability of excited levels populated in the $\beta$ decay. More details on the way the analysis is performed can be found elsewhere [40,41].

So far, two segmented TAS have been developed by the Valencia/Surrey research groups and exploited by the European TAGS collaboration already during two campains of measurement first at the IGISOL facility of the University of Jÿvaskÿla in 2009 and then after the upgrade of IGISOL in 2014.

For the first campain, Rocinante, the Valencia-Surrey Total Absorption Spectrometer was used [42]. It consists in a cylindrical 12-fold segmented $\mathrm{BaF}_{2}$ detector with a length and external diameter of $25 \mathrm{~cm}$, and a longitudinal hole of $5 \mathrm{~cm}$ diameter. It was coupled to a $0.5 \mathrm{~mm}$ thick Si detector with a $\beta$-detection efficiency of about $25 \%$ to get rid of the backgrounds. The setup offers a total efficiency of more than $80 \%$ up to $10 \mathrm{MeV}$. At that period, 7 nuclei of interest for decay heat purpose (including $4 \beta$-delayed emitters) have been measured and published $[7,33,42]$. Their impacts on the decay heat and antineutrino energy spectra calculations have partly been presented and discussed in Sections 2.1 and 2.2.2 of these proceedings.

For the second campain, the new Decay Total Absorption $\gamma$-ray Spectrometer (DTAS) has been successfully installed and used [43]. It consists in 18 modules of $\mathrm{NaI}(\mathrm{Tl})$ crystals. The DTAS spectrometer was completed with a HPGe detector. During this campain, 12 nuclei of Subatech Nantes team proposal of direct interest for antineutrino spectra predictions associated to 11 nuclei of Valencia team proposal of direct interest for decay heat were measured. The whole set of nuclei are currently under study or in the process of being published in the course of $2018[44,45]$. They will allow to update or complete nuclear databases with a list of nuclei henceforth Pandemonium free.

\subsection{Beta-delayed neutron emission properties, a "by-product" of TAS measurements}

Concerning BDN, even if the TAGS measurements are not dedicated to direct measurement of neutrons, they can bring indirect information on some parameters of the properties of $\beta$-decays with delayed neutron emission. As an illustration, the 1st TAGS campain at the JYFL has allowed to evidence an enhanced $\gamma$ emission above the neutron separation threshold in several nuclei. These emissions are not reproduced by the statistical model. These results have implications on nuclear astrophysics processes such as the r-process, as the ratio of $\gamma$ to neutron widths used in the calculation of neutron capture cross sections can be extracted from the $\beta$ decay data measurements [46].

The use of TAGS data in complement to neutron measurements $\left(P_{n}\right.$ values) brings a valuable cross-check to ensure that there is no inconsistensies in the $\beta$ decay properties of fission products in databases.

\subsection{Recent new bias in nuclear data}

Quite recently in a NEA/WPEC25 meeting, large discrepancies have been pointed-out between the mean $\gamma$ energies associated to the $\beta$-decay of several nuclei deduced from TAGS Greenwood and Rudstam [4,47]. This point has been recently investigated by the TAGS Valencian's group who made a careful comparison of these mean energies between the data Pandemonium free from Greenwood and their own measurements, and the data measured by Tengblad/Rudstam [42]. This study is summarized in Figure 4 where the difference between the mean $\gamma$ energies of (Greenwood/Valencia) and Rudstam is depicted as a function of the $Q_{\beta}$ for 30 nuclei.

The comparison shows an uncertainty of the mean energy of $50 \%$ in many cases. Actually, the decay of ${ }^{91} \mathrm{Rb}$ which was assumed to be Pandemonium free by Rudstam et al. was used at the time of the measurements as a normalization point in their systematic studies. After applying a new normalization based on their own measurement of the ${ }^{91} \mathrm{Rb}$ on the results of Rudstam et al., Rice et al. show that even if the mean value of the differences of the mean $\gamma$ energies is reduced from $-360 \mathrm{keV}$ to $-180 \mathrm{keV}$, there is a remaining systematic difference between the two sets of data. Moreover, they still obtain a large spread of the observed differences. This example illustrates once more the necessity to multiply experiments to complete or correct nuclear databases as much as possible. One can also wonder how such kind of discrepancies can impact the evaluation of nuclei and indirectly the decay heat determination (or other applications). 


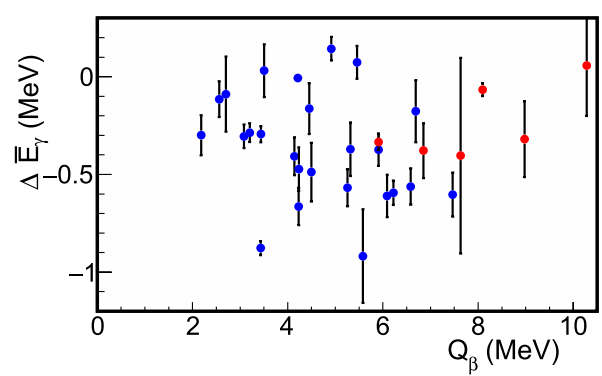

Fig. 4. Differences between the mean energies reported in the work of Rudstam et al. and the deduced mean $\gamma$ energies from the work of Greenwood et al. (blue points) and from the work of the TAGS Valencia/Nantes's groups (red points) [42].

\section{Conclusions and perspectives}

In these proceedings, we have shown that measurements of many nuclei of interest for decay heat, antineutrinos, and a few $\beta$-delayed $n$ emitters have been performed during the past years or are programmed in a near future using the Total Absorption $\gamma$-ray Spectroscopy technique. This direction still needs to be followed as it is mandatory to better characterize fission product fundamental $\beta$-decay properties and especially to improve NDB and all the subsequent applications. The results of the second TAGS campaign in Jyväskylä are already partly available and will be completed in 2018. We then expect to assess the overall impact of all the TAS measurements performed during the last decade on decay heat, reactor antineutrinos and $\beta$ delayed neutron observables by end 2018. Some effort will also be dedicated to include the new measured data in generation IV reactor simulations. The next step regarding the summation method calculations for both $\mathrm{DH}$ and antineutrinos will be the adjunction of uncertainties on the predictions. A proper propagation of uncertainties including FY correlations and decay data correlations is mandatory for that purpose. This task is very challenging since no covariance matrices associated to the decay data exist and the methodology to obtain the covariance matrices of the fission yields is still debated. In addition models are still needed for several tens of nuclei and the shape of forbidden non-unique transitions is not well under control impacting the reactor antineutrino calculations as well as nuclear structure and astrophysics. Solving the issues listed above will require to bring together experimental, simulation, evaluation and theoretical activities.

Several results obtained by the European TAGS collaboration have been used to illustrate the authors statements in these proceedings. The authors would like to acknowledge their colleagues from the TAGS collaboration. The authors acknowledge the nuclear data section of the IAEA and especially Paraskevi (Vivian) Dimitriou for fostering research in the field through the TAGS consultant meetings and the CRP about $\beta$-delayed neutron emission. This work was supported by the CHANDA European project, the In2p3 institute of CNRS, and the NEEDS challenge through the NACRE project.

\section{Author contribution statement}

All the authors have contributed to the scientific content of the paper and approved the final manuscript.

\section{References}

1. J.C. Hardy et al., Phys. Lett. B 71, 307 (1977)

2. M. Estienne et al., Nucl. Data Sheets 120, 149 (2014)

3. M. Fleming, J.-C. Sublet, report 2015 Fispact II, CCFE-R (15) 28

4. T. Yoshida et al., OECD/NEA Working Party for International Evaluation Co-operation, Vol. 1425 (2007) 25. Nuclear Science NEA/WPEC-25 (2007)

5. K. Takahashi, M. Yamada, T. Kondoh, At. Data Nucl. Data Tables, 12, 101 (1973)

6. T. Yoshida, R. Nakasima, J. Nucl. Sci. Technol. 18, 393 (1981)

7. A. Algora et al., Phys. Rev. Lett. 105, 202501 (2010)

8. A. Tobias, Technical report, Central Electricity Generating Board, 1989

9. M. Chadwick et al., Nucl. Data Sheets 112, 2887 (2011)

10. A. Koning et al., Technical Report NEA No. 6190, OECD NEA, 2006

11. K. Shibata et al., J. Nucl. Sci. Technol. 48, 1 (2011)

12. K.-H. Schmidt, B. Jurado, C. Amouroux, General view on nuclear fission (2014), pp. 1-208, in2p3-00976648, www.khserzhausen.de/GEFY.html

13. J.K. Dickens et al., Technical Report ORNL/NUREG-14, Oak Ridge National Laboratory, 1979

14. S. Li, Beta Decay Heat Following 235U, 238U and $239 \mathrm{Pu}$ Neutron Fission, Ph.D. thesis, University of Massachusetts Lowell, Department of Physics, 1997

15. H.V. Nguyen, Gamma-ray Spectra and Decay Heat Following 235U Thermal Neutron Fission, Ph.D. thesis, University of Massachusetts Lowell, Department of Physics, 1997

16. E.H. Seabury, Gamma-ray Decay Heat Measurements Following $238 \mathrm{U}(\mathrm{n}, \mathrm{f})$ and $239 \mathrm{Pu}(\mathrm{n}, \mathrm{f}), \mathrm{Ph}$.D. thesis, University of Massachusetts Lowell, Department of Physics, 1997

17. J.-C. Benoît, Développement d'un code de propagation des incertitudes des données nucléaires sur la puissance résiduelle dans les réacteurs à neutrons rapides, $\mathrm{PhD}$ thesis, University of Paris Sud, CEA, 2012

18. J. Katakura, J. Nucl. Sci. Technol. 50, 99 (2013)

19. Y. Abe et al. (Double Chooz Collaboration), Phys. Rev. Lett. 108, 131801 (2012)

20. F.P. An et al. (Daya Bay Collaboration), Phys. Rev. Lett. 108, 171803 (2012)

21. J.K. Ahn et al. (RENO Collaboration), Phys. Rev. Lett. 108, 191802 (2012)

22. Th.A. Mueller et al., Phys. Rev. C 83, 054615 (2011)

23. G. Mention, M. Fechner, Th. Lasserre, Th. Mueller, D. Lhuillier, M. Cribier, A. Letourneau, Phys. Rev. D 83, 073006 (2011)

24. K.N. Abazajian et al., (2012), arXiv:1204.5379

25. F.P. An et al. (Daya Bay Collaboration), Phys. Rev. Lett. 118, 251801 (2017)

26. M. Fallot, Viewpoint: Getting to the Bottom of an Antineutrino Anomaly, Physics 10, 66 (2017)

27. F. An et al. (Juno collaboration), J. Phys. G 43, 030401 (2016) 
28. L.A. Mikaelian, Proc. Int. Conf. Neutrino-77, v. 2(1977), p. 383

29. P. Dimitriou, A.L. Nichols, IAEA report INDC(NDS)-0676, IAEA, Vienna, Austria, 2015

30. M. Fallot et al., Phys. Rev. Lett. 109, 202504 (2012)

31. MURE, MCNP utility for reactor evolution (2009), http:// www.nea.fr/tools/abstract/detail/nea-1845, O. Meplan, Technical Reports No. LPSC 0912 and No. IPNO-09-01, 2009 32. P. Huber, Phys. Rev. C 84, 024617 (2011)

33. A.-A. Zakari-Issoufou et al., Phys. Rev. Lett. 115, 102503 (2015)

34. A.A. Sonzogni, T.D. Johnson, E.A. McCutchan, Phys. Rev. C 91, 011301(R) (2015)

35. D.A. Dwye, T.J. Langford, Phys. Rev. Lett. 114, 012502 (2015)

36. https://nucleus.iaea.org/sites/connect-members/bDN/

Pages/default.aspx
37. D. Foligno, P. Leconte, EPJ Nuclear Sci. Technol. 4, 31 (2018)

38. A. Algora et al., Nucl. Phys. A 654, 727c (1999)

39. Z. Hu et al., Phys. Rev. C 60, 024315 (1999)

40. D. Cano-Ott et al., Nucl. Instr. Meth. A 430, 488 (1999)

41. D. Cano-Ott et al., Nucl. Instr. Meth. A 430, 333 (1999)

42. S. Rice et al., Phys. Rev. C 96, 014320 (2017), arXiv:1704.01915

43. V. Guadilla et al., Nucl. Inst. Meth. B 376, 334 (2016)

44. V. Guadilla, TAGS measurements for neutrino physics and applications, Ph.D. thesis, University of Valencia, 2017

45. L. Le Meur, Etude des propriétés de désintégration bêta de noyaux exotiques à l'aide de la technique de Spectroscopie par Absorption Totale (TAS), Ph.D. thesis, University of Nantes, in preparation

46. J.L. Tain et al., Phys. Rev. Lett. 115, 062502 (2015)

47. A.L. Nichols et al., INDC(NDS)-0499, 2006

Cite this article as: Magali Estienne, M. Fallot, L. Giot, L. Le Meur, A. Porta, Recent advances in beta decay measurements, EPJ Nuclear Sci. Technol. 4, 24 (2018) 